\title{
Role of Target Resonances In Low-energy nucleon and $\alpha$ Interactions with Weakly-bound Nuclei.
}

\author{
Ken Amos* and Steven Karataglidis \\ Department of Physics, University of Johannesburg, P.O. Box 524, Auckland Park, South Africa \\ School of Physics, University of Melbourne, Victoria 3010, Australia \\ E-mail: amos@physics.unimelb.edu.au; stevenka@uj.ac.za
}

Dirk van der Knijff

School of Physics, University of Melbourne, Victoria 3010, Australia

E-mail: dvanderknijffegmail.com

\section{Paul Fraser, Kym Massen-Hane, Igor Bray, and Alisher Kadyrov}

Department of Physics, Astronomy and Medical Radiation Sciences, Curtin University, GPO Box U1987, Perth 6845, Australia

E-mail paul.fraser@curtin.edu.au; A.Kadyrov@curtin.edu.au;

kym.massen-haneestudent.curtin.edu.au; igor.bray@curtin.edu.au

\section{Luciano Canton}

Istituto Nazionale di Fisica Nucleare, sezione di Padova, Padova I-35131, Italia

E-mail: luciano.cantonepd.infn.it

\section{Ruben Fossión}

Instituto de Ciencias Nucleares, Universidad Nacional Autónoma de México, 04510, Ciudad de México, Mexico

E-mail:ruben.fossionenucleares.unam.mx

\section{Juris Svenne}

Department of Physics and Astronomy, University of Manitoba, and Winnipeg Institute for Theoretical Physics, Winnipeg, Manitoba, Canada R3T 2N2

E-mail:svenneephysics.umanitoba.ca

\begin{abstract}
Radioactive ion beams of weakly bound nuclei are now being used to investigate properties of nuclear systems well off of the valley of stability. Those ions can and do have low excitation spectra that involve particle unstable resonances. We seek to describe the spectra of the compound systems formed, and the low energy scattering cross sections for clusters formed coupling a nucleon or an $\alpha$-particle with such weakly bound systems.
\end{abstract}

The 26th International Nuclear Physics Conference

11-16 September, 2016

Adelaide, Australia

${ }^{*}$ Speaker. 


\section{Introduction}

We have investigated the role of target states being resonances by developing a method in which those resonance characteristics can be taken into account in coupled-channel descriptions of the compound systems formed by those nuclei with nucleons and with $\alpha$-particles. The method is an adaptation of a multi-channel algebraic scattering (MCAS) theory with which coupled-channel Lippmann-Schwinger equations for the clusters of a nucleon, or an $\alpha$-particle, and a nucleus can be solved. Using MCAS, the spectra (bound and resonant) of the compound system can be found as can the scattering cross sections from the target nucleus or, by inverse kinematics, of a radioactive ion from hydrogen or an $\alpha$-particle.

\section{Theory}

The MCAS method [1, 2] solves coupled channel Lippmann-Schwinger equations for a chosen two-body system. The theory is built upon a sturmian expansion of the given choice of interaction matrix of potential functions for the system. The set of sturmians that form the basis of the expansions are determined from the self-same matrix of potentials and, if necessary, can be selected to ensure that the Pauli principle is not violated. The approach, most suited to deal with low energies, is noteworthy because its formulation facilitates a systematic determination of all sub-threshold bound states and compound resonances within any energy range considered.

While the required starting matrix of potentials may be constructed from any model of nuclear structure, to date we have used just a simple collective rotation model to define those potentials allowing deformation therein to second order. We start with coordinate space potentials which for a nucleon-nucleus system has the basic form

$$
V_{c c^{\prime}}(r)=f(r)\left\{V_{0} \delta_{c c^{\prime}}+V_{l l}[\ell \cdot \ell]_{c c^{\prime}}+V_{s s}[\mathbf{s} \cdot \mathbf{I}]_{c c^{\prime}}\right\}+\frac{1}{r} \frac{d f(r)}{d r} V_{l s}[\ell \cdot \mathbf{s}]_{c c^{\prime}}
$$

for each channel (c) where $\mathrm{c}$ denotes a unique set of quantum numbers. A deformed Woods-Saxon form has been used for the function $f(r)$. A similar interaction but with different operators is used in the $\alpha$-nucleus case.

To consider nucleon and $\alpha$-particle interactions with a (light mass) nuclei, we suppose that the collective (rotational) model describes the 'target' nucleus. Without additional features, solutions of coupled-channel equations built from local interactions can involve violation of the Pauli principle with the extra core particle being allowed to occupy already occupied orbits [1]. That can be prevented by including orthogonalising pseudo-potentials (OPP) [3] in the Hamiltonian. By solving the Lippmann-Schwinger equations in momentum space, the method defines both the bound (to particle emission) and scattering states of the compound nucleus. Bound states of the compound system can be found using negative energies when all channels are closed. For positive energies, to systematically identify all resonance structures of the compound system, we use a spectral representation of the $T$-matrix in terms of complex sturmian eigenvalues.

Sets of sturmian functions, $\Phi_{c^{\prime} n}(r)$ and their eigenvalues $\eta_{n}$, are determined from the coupledchannel interactions, $V_{c c^{\prime}}(r)$. For practical reasons we choose a set $(n)$ of finite rank, with entries being those having largest magnitudes of $\eta_{n}$. These are used to define the form factors in the 
expansion of the same coupled-channel interactions. The form factors, in momentum space, $\hat{\chi}_{c n}(p)$, are the Fourier-Bessel transforms of

$$
\chi_{c n}(r)=\sum_{c^{\prime}=1} V_{c c^{\prime}}(r) \Phi_{c^{\prime} n}(r)
$$

The sturmian eigenvalues, $\eta_{i}$ are found using the Green's function

$$
\begin{aligned}
& {\left[\mathbf{G}_{0}\right]_{n n^{\prime}}=\mu } {\left[\sum_{c=1}^{\text {open }} \int_{0}^{\infty} \hat{\chi}_{c n}(x) \frac{x^{2}}{k_{c}^{2}-x^{2}+i \varepsilon} \hat{\chi}_{c n^{\prime}}(x) d x-\sum_{c=1}^{\text {closed }} \int_{0}^{\infty} \hat{\chi}_{c n}(x) \frac{x^{2}}{h_{c}^{2}+x^{2}} \hat{\chi}_{c n^{\prime}}(x) d x\right], } \\
& k_{c}=\sqrt{\mu\left(E-\varepsilon_{c}\right)} ; h_{c}=\sqrt{\mu\left(\varepsilon_{c}-E\right)}
\end{aligned}
$$

being wave numbers. $\varepsilon_{c}$ is the target state centroid and $E$ is the 'projectile' energy. With these form factors, the momentum space expansion of the non-local interaction matrices is

$$
V_{c c^{\prime}}(p, q)=\sum_{n} \hat{\chi}_{c n}(p) \frac{1}{\eta_{n}} \hat{\chi}_{c^{\prime} n}(q)
$$

and the $T$-matrix follows as

$$
T_{c c^{\prime}} \propto \sum_{n n^{\prime}} \sqrt{k_{c}} \hat{\chi}_{c n}\left(k_{c}\right)\left(\left[\eta-\mathbf{G}_{\mathbf{0}}\right]^{-1}\right)_{n n^{\prime}} \hat{\chi}_{c^{\prime} n^{\prime}}\left(k_{c^{\prime}}\right) \sqrt{k_{c^{\prime}}} .
$$

Here $[\eta]_{n n^{\prime}}=\eta_{n} \delta_{n n^{\prime}}$. The spectrum of the compound system is found from properties of the resolvent in the $T$-matrix, i.e. $\left[\eta-\mathbf{G}_{\mathbf{0}}\right]^{-1}$.

With systems such as $n+{ }^{12} \mathrm{C}$ forming ${ }^{13} \mathrm{C}$, a Hamiltonian has been found [1] which, when used in MCAS evaluations of the $T$-matrices, leads to a very good representation of the spectrum of ${ }^{13} \mathrm{C}$ (bound and resonant to over $4 \mathrm{MeV}$ incident energy). Also the measured scattering cross section in that low energy range was well reproduced. An example is given later. But in the low-energy and low-mass regime where compound-system resonances are important, it is appropriate to take particle instability of target states into account. In basic form [4], this has been done by replacing the target state energy in the resolvent, $\varepsilon_{c} \rightarrow \varepsilon_{c}+i \frac{1}{2} \Gamma_{c}$, so that the Green's functions become

$$
\begin{aligned}
{\left[\mathbf{G}_{0}\right]_{n n^{\prime}}=\mu\left[\sum_{c=1}^{\text {open }} \int_{0}^{\infty} \hat{\chi}_{c n}(x) \frac{x^{2}\left[k_{c}^{2}-x^{2}-i \frac{\mu}{2} \Gamma_{c}\right]}{\left[k_{c}^{2}-x^{2}\right]^{2}+\frac{\mu^{2}}{2} \Gamma_{c}^{2}} \hat{\chi}_{c n^{\prime}}(x) d x\right.} \\
\\
\left.-\sum_{c=1}^{\text {closed }} \int_{0}^{\infty} \hat{\chi}_{c n}(x) \frac{x^{2}\left[h_{c}^{2}+x^{2}+i \frac{\mu}{2} \Gamma_{c}\right]}{\left[h_{c}^{2}+x^{2}\right]^{2}+\frac{\mu^{2}}{2} \Gamma_{c}^{2}} \hat{\chi}_{c n^{\prime}}(x) d x\right] .
\end{aligned}
$$

This equation has no poles on the real axis, and integration may proceed normally. But the target state widths of the Lorentz form do not vanish at $E=0$ and that can lead to non-physical attributes in the evaluated compound spectra and scattering cross sections. The energies of bound states can also be affected causing some to have spurious widths.

To overcome these non-physical behaviours, a scaling factor is applied to target-state widths [5], so that the target states are described using $\varepsilon_{c} \rightarrow \varepsilon_{c}+i \frac{1}{2} U(E) \Gamma_{c}$ where minimum conditions are to be placed the scaling function, namely

$$
U(E=0)=0 ; \frac{d U(E \rightarrow+0)}{d E} \rightarrow 0 ; U\left(\varepsilon_{c}\right)=1 ; U(E \rightarrow \infty)=0 .
$$


The first two conditions eliminate spurious behaviour in the reaction cross sections at low energies.

The use of energy-dependent widths necessitates an energy-dependent addition to the targetstate centroid, and so changing the energy factor in the resolvent of the Green's functions as $\varepsilon_{c} \rightarrow \varepsilon_{c}+\Delta \varepsilon_{c}(E)+i \frac{1}{2} U(E) \Gamma_{c}$. This extension is needed because the Green's functions define the sturmian eigenvalues of the expansion of the potential, thus making the interactions involving the target states complex. In effect they become optical potentials. Energy-dependent imaginary components in optical potentials lead to wave equations that violate causality unless the potentials are constrained by addition of a dispersion-relation correction.

With MCAS, the dispersion relation is an energy-dependent adjustment of the target-state centroid energy, $\Delta \varepsilon_{c}(E)$, given by the principal part integral,

$$
\Delta \varepsilon_{c}(E)=\frac{\Gamma_{c}}{2} \frac{1}{\pi} \mathscr{P} \int_{0}^{\infty} \frac{U\left(E^{\prime}\right)}{E^{\prime}-E} d E^{\prime} .
$$

The wave numbers of Eq. (2.3) when target states are resonances are given by

$$
k_{c}=\sqrt{\mu\left(E-\varepsilon_{c}-\Delta \varepsilon_{c}(E)\right)} ; h_{c}=\sqrt{\mu\left(\varepsilon_{c}+\Delta \varepsilon_{c}(E)\right)-E} .
$$

One candidate for an energy-dependent target-state width scaling is a Wigner distribution,

$$
U(E)=e^{m}\left(\frac{E}{\varepsilon_{c}}\right)^{n} e^{-m\left(\frac{E}{\varepsilon_{c}}\right)^{n}} \mathscr{H}(E) .
$$

With $m$ and $n$ positive integers, this meets the necessary conditions. The Heaviside function ensures proper bound-state properties since, without it, the Green's functions are complex for $E \leq 0$.

More details of this method and applications are given in a recent publication [6].

\section{Results}

\subsection{A gedanken case; $n+{ }^{12} \mathrm{C}$}

Past MCAS studies gave very good representation of the bound and low excitation resonant structure of ${ }^{13} \mathrm{C}$ as illustrated in Fig. 1. In those MCAS calculations, three states of ${ }^{12} \mathrm{C}$ were taken into account. They are the ground $0^{+}$, the $2_{1}^{+}(4.44 \mathrm{MeV})$, and the $0_{2}^{+}(7.76 \mathrm{MeV})$ states. Those states all lie below and particle emission threshold so the results shown do not involve target states having widths. Gedanken calculations have been made taking the $2_{1}^{+}$and $0_{2}^{+}$states to be resonances. The effect of introducing particle emissive widths to these states immediately affects results, especially reaction cross sections, as seen in Fig. 2. With no resonance character in the target states the reaction cross section becomes non-zero only above the energy of the first excited target state, at $4.81 \mathrm{MeV}$ (lab. frame). When target-state widths are allowed the reaction cross section becomes non-zero for all projectile energies, with structure increasing with the small width values shown. With larger widths the reaction cross section degenerates to a smoothly rising form (from $E=0$ ) having a broader shape to the resonance in the $4-5 \mathrm{MeV}$ region. In all cases, the reaction cross section vanishes smoothly as $E \rightarrow 0$ provided the causality conditions are met.

Now we consider target nuclei that have particle emissive resonant states in their low-lying spectrum. Specifically we have used ${ }^{8} \mathrm{Be}$ and ${ }^{6} \mathrm{He}$ as targets in MCAS cluster model calculations. The low lying spectra of these two nuclei are shown in Fig. 3. With each, the lowest three states have been used in MCAS evaluations. 


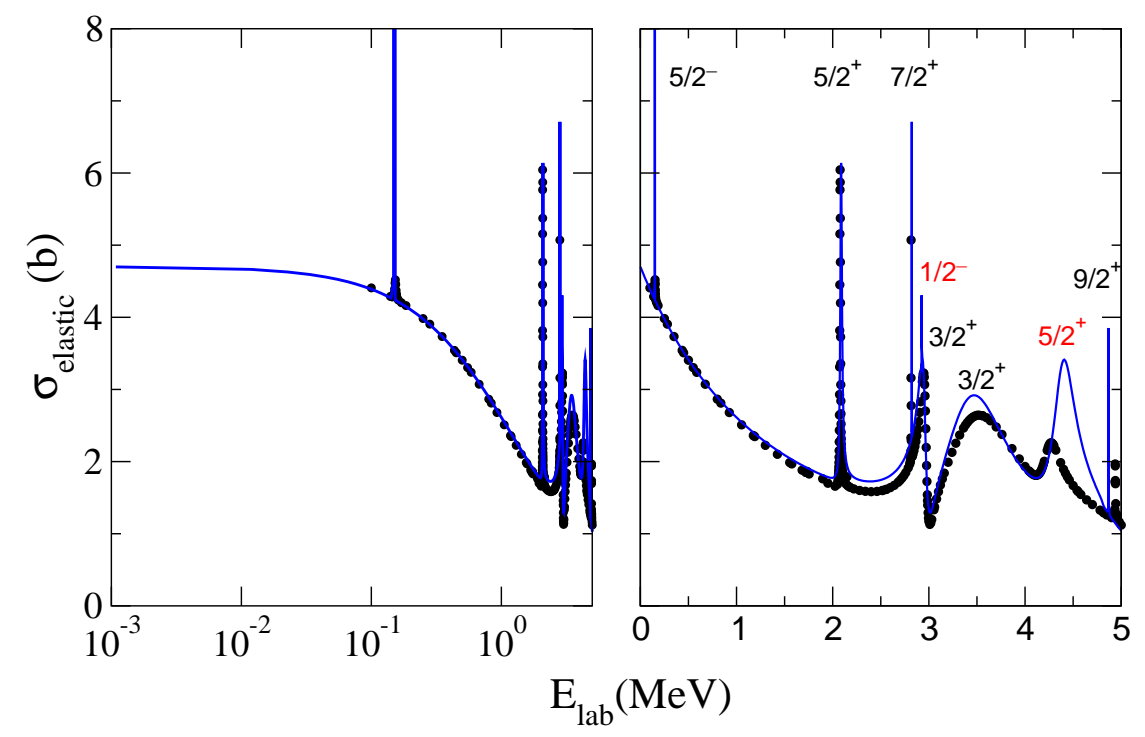

Figure 1: Low energy $n+{ }^{12} \mathrm{C}$ scattering shown on logarithmic (left) and linear (right) scales of energy. Spin-parities of states listed; red values interchanged in experimental list.

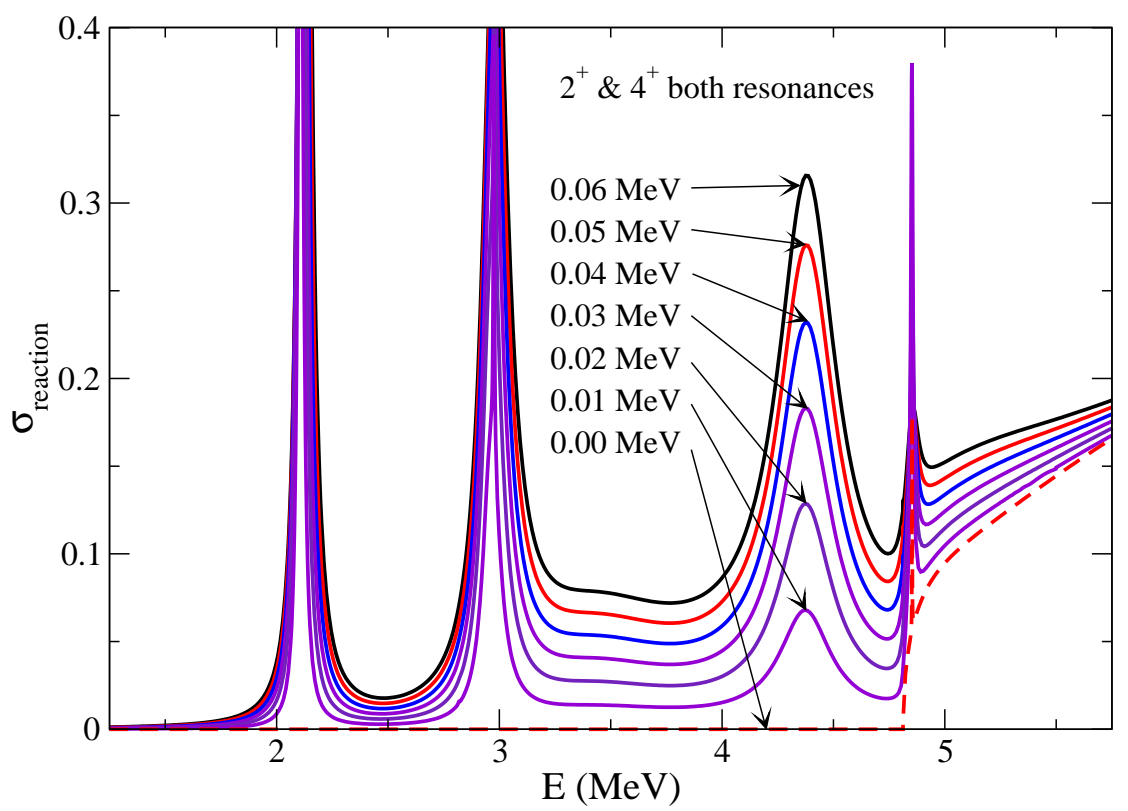

Figure 2: Low energy $n+{ }^{12} \mathrm{C}$ reaction scattering cross sections when the excited states are resonances.

\subsection{Results for the $n+{ }^{8} \mathrm{Be}$ system}

With a model interaction that gives a reasonable match to the five lowest states in the spectrum of ${ }^{9} \mathrm{Be}$, the MCAS cross sections for $n-{ }^{8} \mathrm{Be}$ scattering are shown in Fig. 4.

By taking the states of ${ }^{8} \mathrm{Be}$ as having no widths, the elastic scattering cross section is very structured showing resonances clearly. The reaction cross section is zero until the first excitation is possible. 


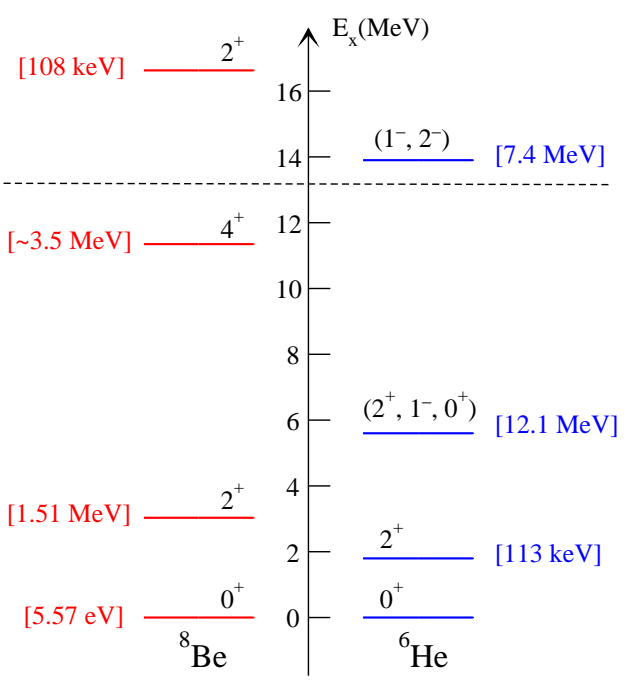

Figure 3: Low energy spectra: ${ }^{8} \mathrm{Be}$ and ${ }^{6} \mathrm{He}$.

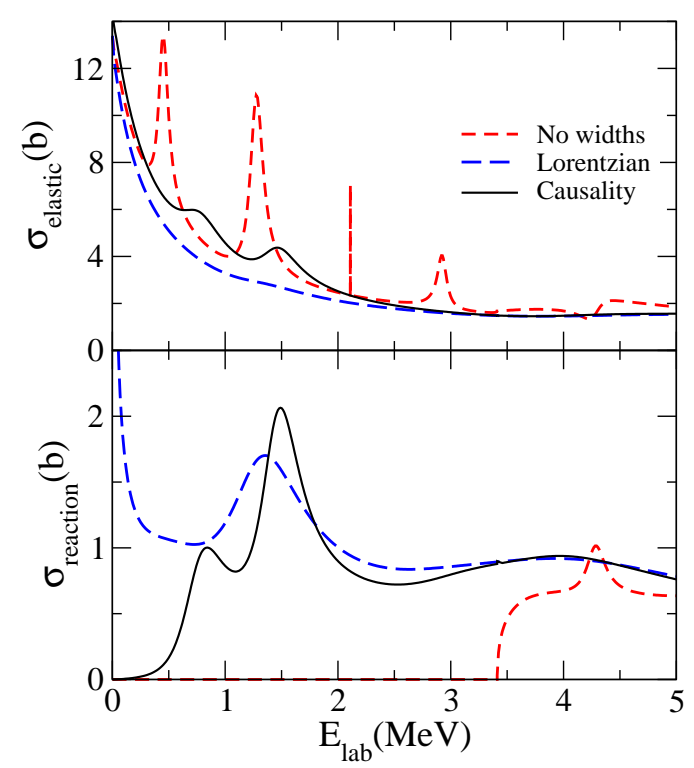

Figure 4: Calculated $n+{ }^{8}$ Be elastic scattering (top) and reaction (bottom) cross sections.
When the (two excited) target states have Lorentzian shapes results change markedly. The elastic scattering cross section now is featureless and the reaction cross section is non-zero from zero projectile energy but at low energies the asymptotic behaviour is unphysical. However, by using the energy weighted scaling factor to ensure causality, while also giving a non-zero reaction cross section from $E=0$, there is no erroneous asymptotic rise in the reaction cross section near threshold. Causality restoration, by altering centroids, affects the shape of the cross sections, with consequences for the parameters required in defining the scattering-potentials.

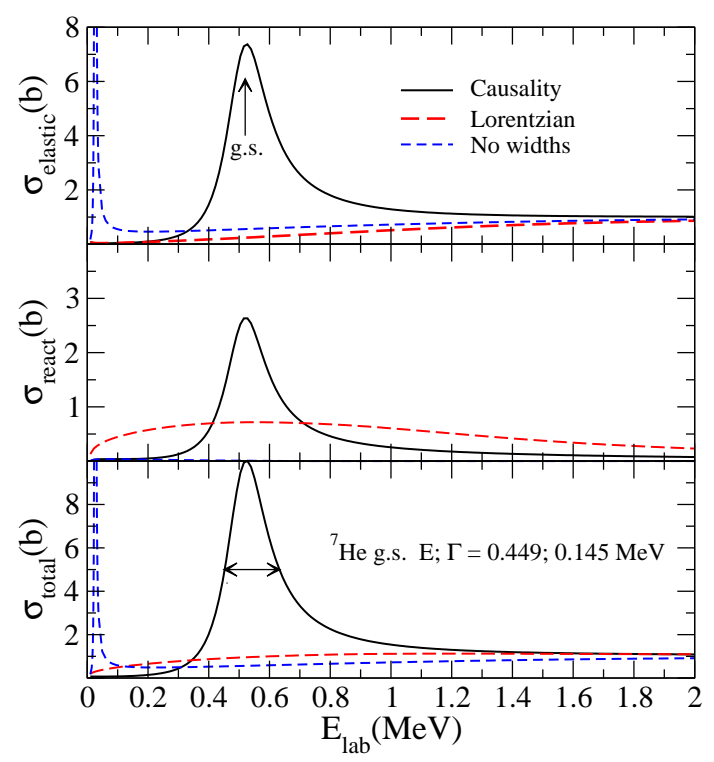

Figure 5: $n+{ }^{6} \mathrm{He}$ scattering cross sections: elastic (top), reaction (middle), and total (bottom).

\subsection{Results for the $n+{ }^{6} \mathrm{He}$ system}

Calculations of $n+{ }^{6} \mathrm{He}$ were made and cross section results are shown in Fig. 5. The interactions were set so that the full (causality) result gave the best match to the (few) known states of the particle missive compound, ${ }^{7} \mathrm{He}$. Using no widths for the (two excited) target states with this interaction made the ground state of the compound almost be bound while using solely Lorentzian forms, which gave a reasonable ground state energy, smoothed out any resonance structure in the cross sections. But with the scaling factor ensuring causality, the cross sections not only gave the ${ }^{7} \mathrm{He}$ ground state resonance centroid correctly but also its width. 


\subsection{Results for the $\alpha-{ }^{6} \mathrm{He}$ system}

MCAS has now been developed to have the $\alpha$-particle as a member of the cluster and an interaction found that leads to the spectra given in Fig. 6 . The threshold for $\alpha$ break-up in ${ }^{10} \mathrm{Be}$ lies

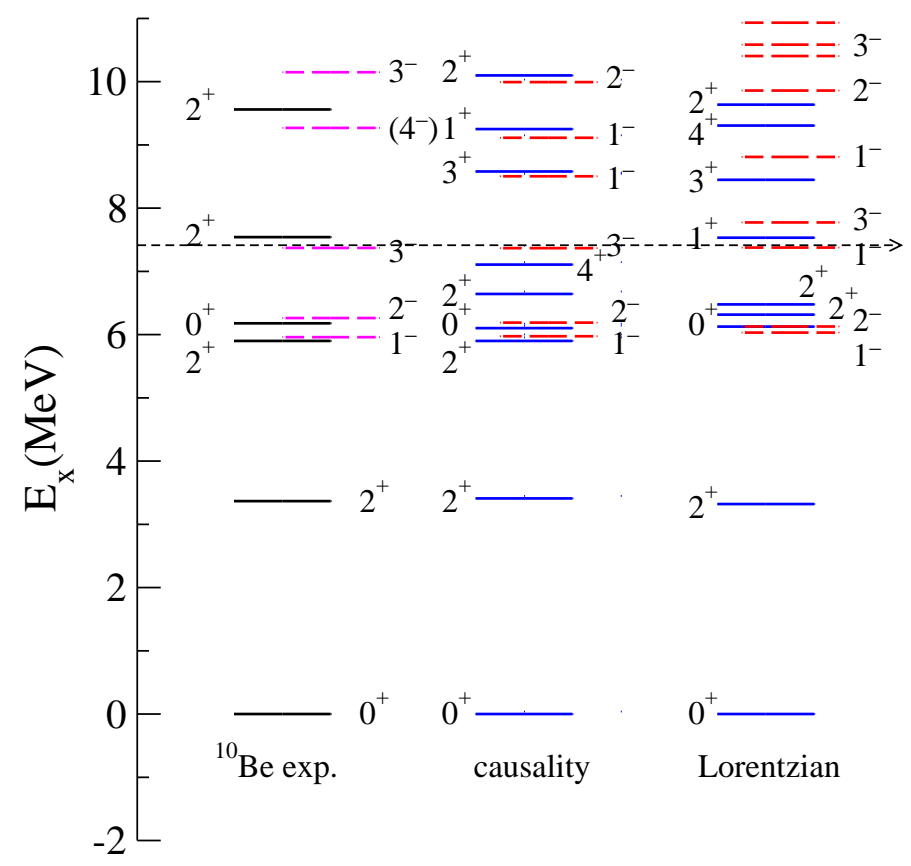

Figure 6: Spectra of ${ }^{10} \mathrm{Be}$ from the $\alpha-{ }^{6} \mathrm{He}$ cluster calculations compared with experiment.

some $7 \mathrm{MeV}$ above its ground. Our MCAS results match the lowest six bound states quite well but give more states in the continuum than observed. Most do not seem to contribute significantly to scattering cross sections however. Data have been obtained using a radioactive ion beam of ${ }^{6} \mathrm{He}$, which, by using inverse kinematics, in Fig. 7 compare favourably with our MCAS calculations.
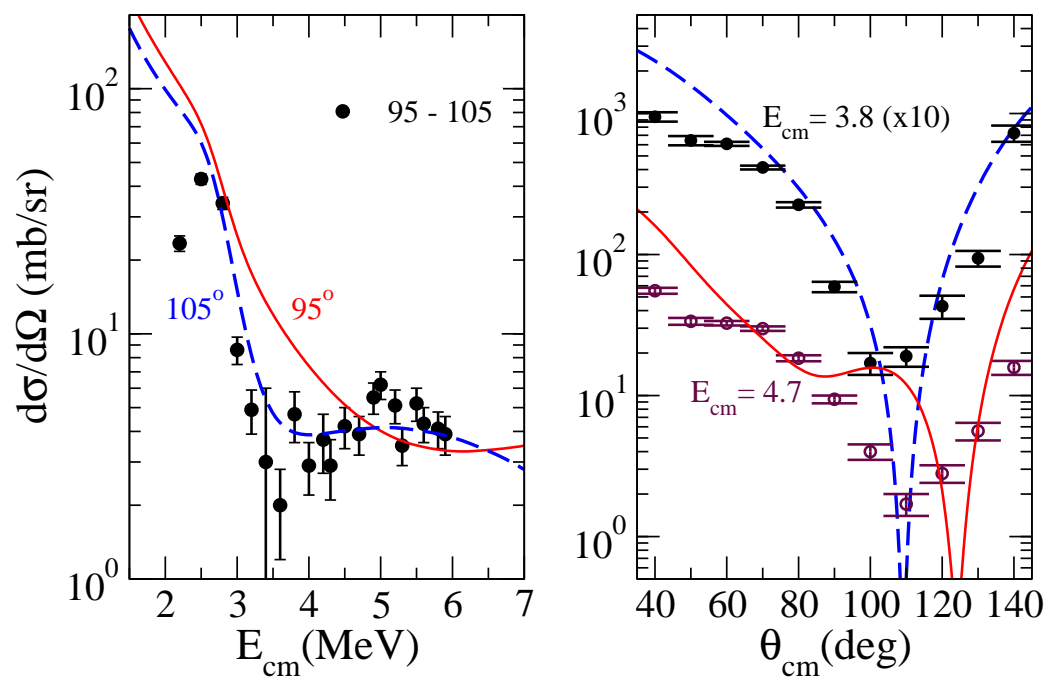

Figure 7: Cross sections from $\alpha_{-}{ }^{6} \mathrm{He}$ scattering calculations compared with experiment. 


\section{Conclusions}

Applications of the MCAS theory have been made to discern the structure of the compound nuclei underlying various di-cluster nuclear systems of light mass. Despite the simplicity of the collective model used to specify the input matrix of interaction potentials, good agreement has been found with available data, even regarding a nucleus lying beyond the proton drip line.

The method used accounts for particle-unstable states in a (target) nucleus undergoing lowenergy resonant scattering. By choosing an appropriate target-state resonance shape modifying the usual Lorentzian shape of a resonance state consistent with physically realistic conditions, results are free of any unphysical behaviour at the scattering threshold. This modification can violate causality but that is restored by using a dispersion relation to define an energy correction.

This method gives scattering cross sections that are markedly different from those found when particle instability (of a target nucleus state) is not considered. Compound-system resonances decrease in magnitude and increase in width, with otherwise narrow resonances becoming obscured into the scattering background. In a case studied improved agreement between calculated and observed widths of resonances in the compound system resulted.

When using parameter-driven scattering potentials, the effects of the target-state resonance shape, and in the case of energy-dependent modified Lorentzians, the correction is to restore causality, are non-trivial. Compound spectra associated with, and scattering cross sections from, weaklybound radioactive ion beams with light-mass targets should be influenced by these considerations.

\section{Acknowledgments}

This work is supported by the Australian Research Council, National Research Foundation of South Africa, and U.S. National Science Foundation under Grant No. PHY-1415656.

\section{References}

[1] K. Amos, L. Canton, G. Pisent, J. P. Svenne, and D. van der Knijff, An algebraic solution of the multichannel problem applied to low energy nucleon-nucleus scattering, Nucl. Phys. A728, 65 (2003).

[2] L. Canton, G. Pisent, K. Amos, S. Karataglidis, J. P. Svenne, and D. van der Knijff, Collective coupling analysis of spectra of mass-7 isobars: ${ }^{7} \mathrm{He},{ }^{7} \mathrm{Li},{ }^{7} \mathrm{Be},{ }^{7} \mathrm{~B}$, Phys. Rev. C 74, 064605 (2006).

[3] L. Canton, G. Pisent, J. P. Svenne, D. van der Knijff, K. Amos, and S. Karataglidis, Role of the Pauli principle in collective-model coupled-channels calculations, Phys. Rev. Lett. 94, 122503 (2005).

[4] P. R. Fraser, K. Amos, L. Canton, G. Pisent, S. Karataglidis, J. P. Svenne, and D. van der Knijff, Coupled-channel evaluations of cross sections for scattering involving particle-unstable resonances, Phys. Rev. Lett. 101, 242501 (2008).

[5] L. Canton, P. R. Fraser, J. P. Svenne, K. Amos, S. Karataglidis, and D. van der Knijff, Energy-dependent target widths in coupled-channel scattering, Phys. Rev. C 83, 047603 (2011).

[6] P. R. Fraser, K. Massen-Hane, K. Amos, I. Bray, L. Canton, R. Fossión, A. S. Kadyrov, S. Karataglidis, J. P. Svenne, and D. van der Knijff, Importance of resonance widths in low-energy scattering of weakly-bound light-mass nuclei, Phys. Rev. C 94, 034603 (2016). 